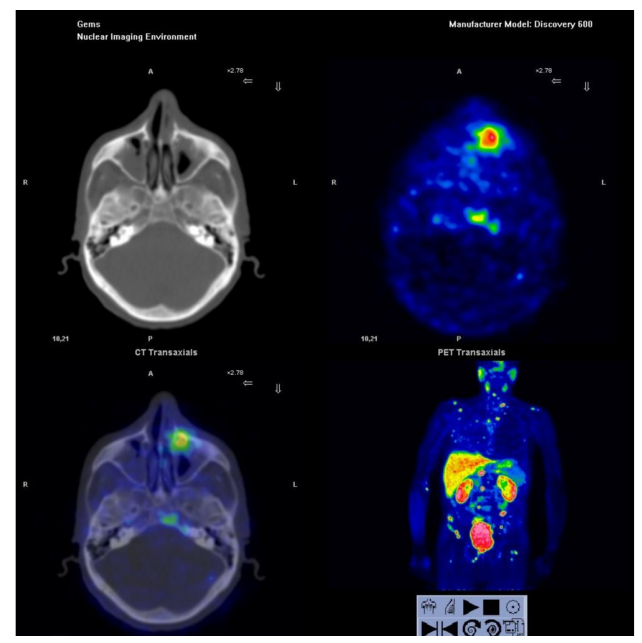

\title{
18F-Choline PET-CT: a potential adjuvant tool for early diagnosis of Osteonecrosis of the Jaw (ONJ) in prostate cancer patients?
}

\author{
Vittorio Fusco ${ }^{1}$, Luigi Tommasi ${ }^{1}$, Alfredo Muni ${ }^{1}$, Antonella Fasciolo ${ }^{1}$, Pamela Francesca Guglielmini ${ }^{1}$, maura vincenti ${ }^{1}$, \\ Daniela Tartara ${ }^{1}$, Paola Brigo
}

1 Azienda Ospedaliera SS. Antonio e Biagio e Cesare Arrigo

Funding: The author(s) received no specific funding for this work.

Potential competing interests: The author(s) declared that no potential competing interests exist.

\section{Abstract}

Background. Nuclear Medicine imaging techniques, i.e., planar bone scan (Tc99 m scintigraphy) and PET (Positron Emission Tomography) with several tracers, have been suggested as useful aids to diagnosis of Osteonecrosis of Jaw $(\mathrm{ONJ})$, that is mostly based on clinical observation (signs and symptoms, with/without bone exposure) and Computed Tomography (CT) scan. Potential advantages of Nuclear Medicine techniques include very early detection of ONJ (due to precocious uptakes), especially in ONJ cases without frank bone exposure. Prostate cancer patients treated with bisphosphonates or denosumab are at risk of ONJ. They undergo bone scan and/or 18F-Choline PET-CT aimed to evaluate response to treatment but attention is generally pointed to extracranial uptakes. Literature data show ability of bone scan to detect maxillary and mandible areas at risk of clinically relevant $\mathrm{ONJ}$, but data are lacking about $18 \mathrm{~F}$ Choline PET-CT.

Material and Methods. We retrospectively reviewed 18F-Choline PET-CT scans of prostate cancer patients with diagnosis of ascertained ONJ (at CT scan) to search for uptakes in maxillary bones and mandible at time of ONJ diagnosis, or before, or after. 
Results. Out of 5 prostate cancer patients undergoing 18F-Choline PET-CT scans before or after the ONJ diagnosis time, 4 had clear jaw uptakes at ONJ site next to ONJ diagnosis time or before; one had no uptake (4 years after ONj diagnosis).

Conclusions. Our limited experience indicates that $18 \mathrm{~F}$-Choline PET-CT scan uptakes, as well as planar bone scan uptakes, are of potential help to early detect ONJ areas, sometimes still in absence of signs and symptoms. Further studies with larger numbers of prostate cancer patients with and without ONJ disease are warranted and should be planned as multicentre trials.

The 18F-Choline PET-CT scan uptakes at maxillary bone and mandible should be reported and underlined by Nuclear Medicine specialists, as well as bone scan uptakes, as an aid to oncologists, oral care specialists and patients.

Background. Nuclear Medicine imaging techniques, i.e., planar bone scan (Tc99 m scintigraphy), SPECT (Single-Photon Emission Computed Tomography) and PET (Positron Emission Tomography) with several tracers (mostly 18F-FDG), have been suggested as potentially useful aids to diagnosis of Osteonecrosis of Jaw (ONJ) in our experience ${ }^{[1][2]}$ and in international literature ${ }^{[3][4][5][6][7][8][9][10][11][12][13][14][15][16][17]}$. ONJ is mostly based on clinical observation (signs and symptoms, with/without bone exposure) and Computed Tomography (CT) scan[18][19][20][21]. A potential advantage of Nuclear Medicine techniques is that of detecting ONJ very early (due to precocious uptakes); this ability might be valuable in patients with suspected ONJ cases (for example patients without frank bone exposure) or even in patients without signs or symptoms but at high risk of ONJ (due to oral risk factors, prolonged antiresorptive treatment, etc. ${ }^{[2][11]}$. Literature data are sound about planar bone scan (99mTc-MDP scintigraphy), SPECT and 18-FDG PET, but are insufficient about 18FCholine PET-CT scan, an exam that recently is largely adopted in metastatic prostate cancer, reducing the prescriptions of Tc99m bone scans in routine clinical practice (due to ability of PET in detecting not only bone metastases but also abnormal lymph nodes and other metastases). Our group reported one case of early uptakes at 18F-Choline PET-CT scan in a prostate cancer patient with multiple-site $\mathrm{ONJ}^{[22]}$. We analysed other cases of $\mathrm{ONJ}$ in prostate cancer patients, to evaluate the potentiality of 18F-Choline PET-CT scan in detecting ONJ sites otherwise studied.

Material and methods. We reviewed clinical data and imaging exams of prostate cancer patients affected by ONJ after denosumab or bisphosphonate treatment and undergoing 18F-Choline PET-CT scan for oncologist's choice to evaluate cancer disease (not jawbone), at the Nuclear Medicine unit of Alessandria Hospital, North-Western Italy.

Results. Imaging exams (CT scan, planar bone scan, SPECT, 18F-Choline PET-CT scan) of 18 prostate cancer patients with ONJ diagnosis, according to SIPMO-SICMF criteria ${ }^{[18][19]}$ were evaluated. Out of 18 patients, 5 patients underwent at least one 18F-Choline PET-CT scan before or after the ONJ diagnosis time and are the object of this preliminary study. Case 1: 77 years, maxillary ONJ after zoledronic acid treatment; one uptake was present at PET three months after the ONJ diagnosis time (clinically and CT positive). (Fig.1) 


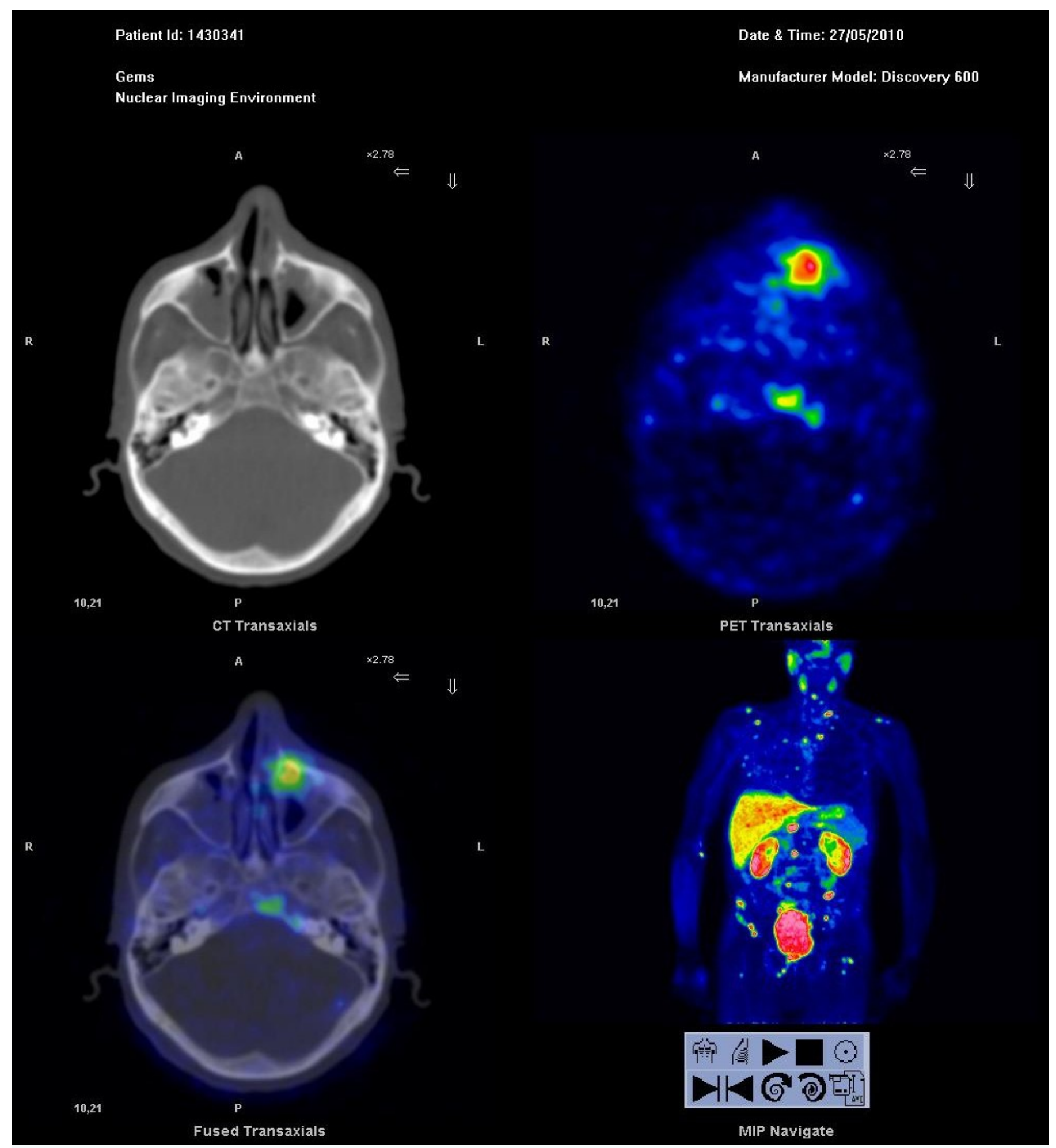

Fig 1. Left maxilla ONJ uptake next to time of ONJ diagnosis.

Case 2. 81 years, mandible ONJ after zoledronic acid treatment; one PET uptake was already present 6 months before definitive ONJ diagnosis, and mostly resolved after two years.(Fig 2 and 3) 


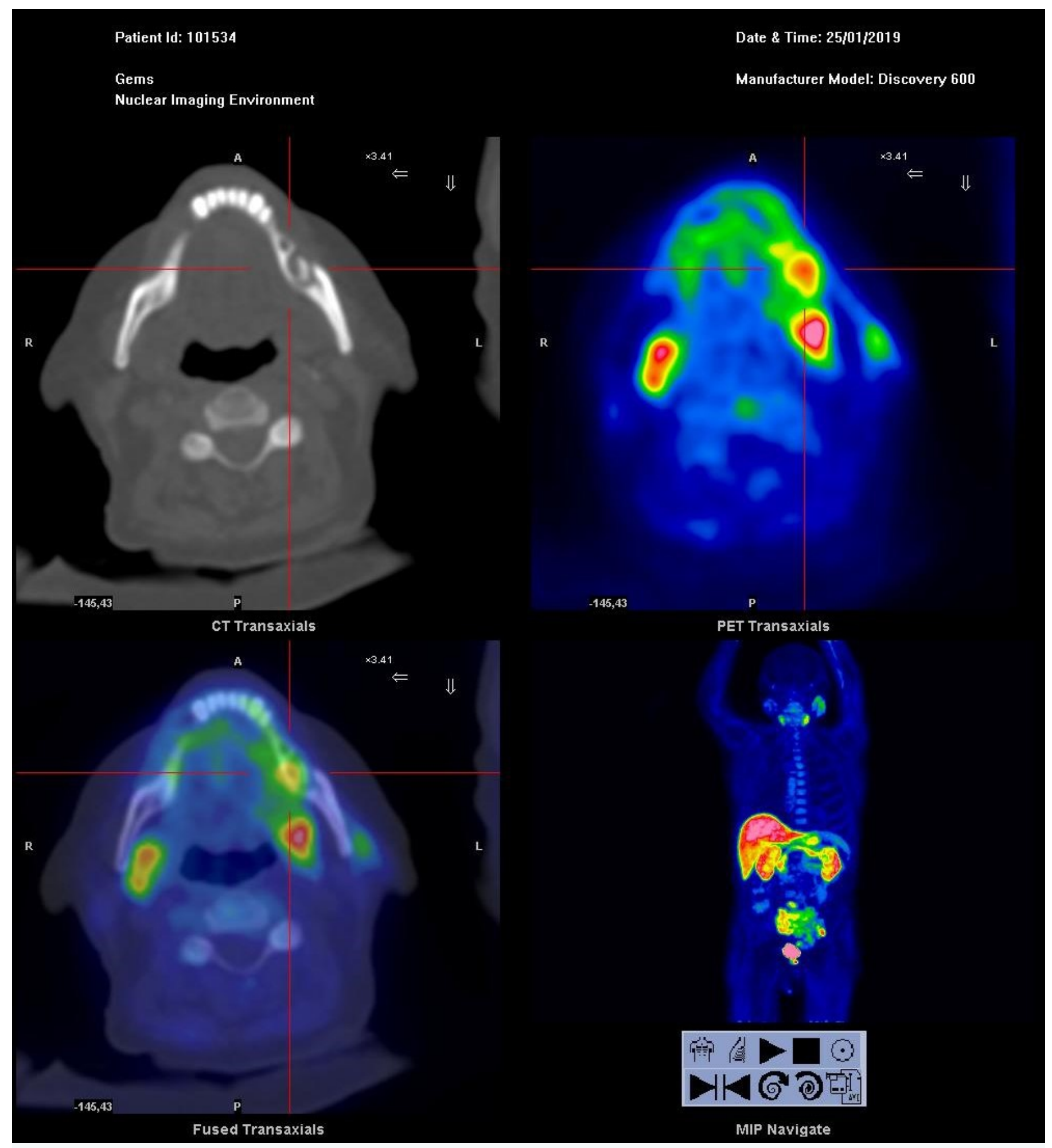

Fig 2 - Left side of mandible ONJ at PET-CT 6 months before ONJ diagnosis time. 


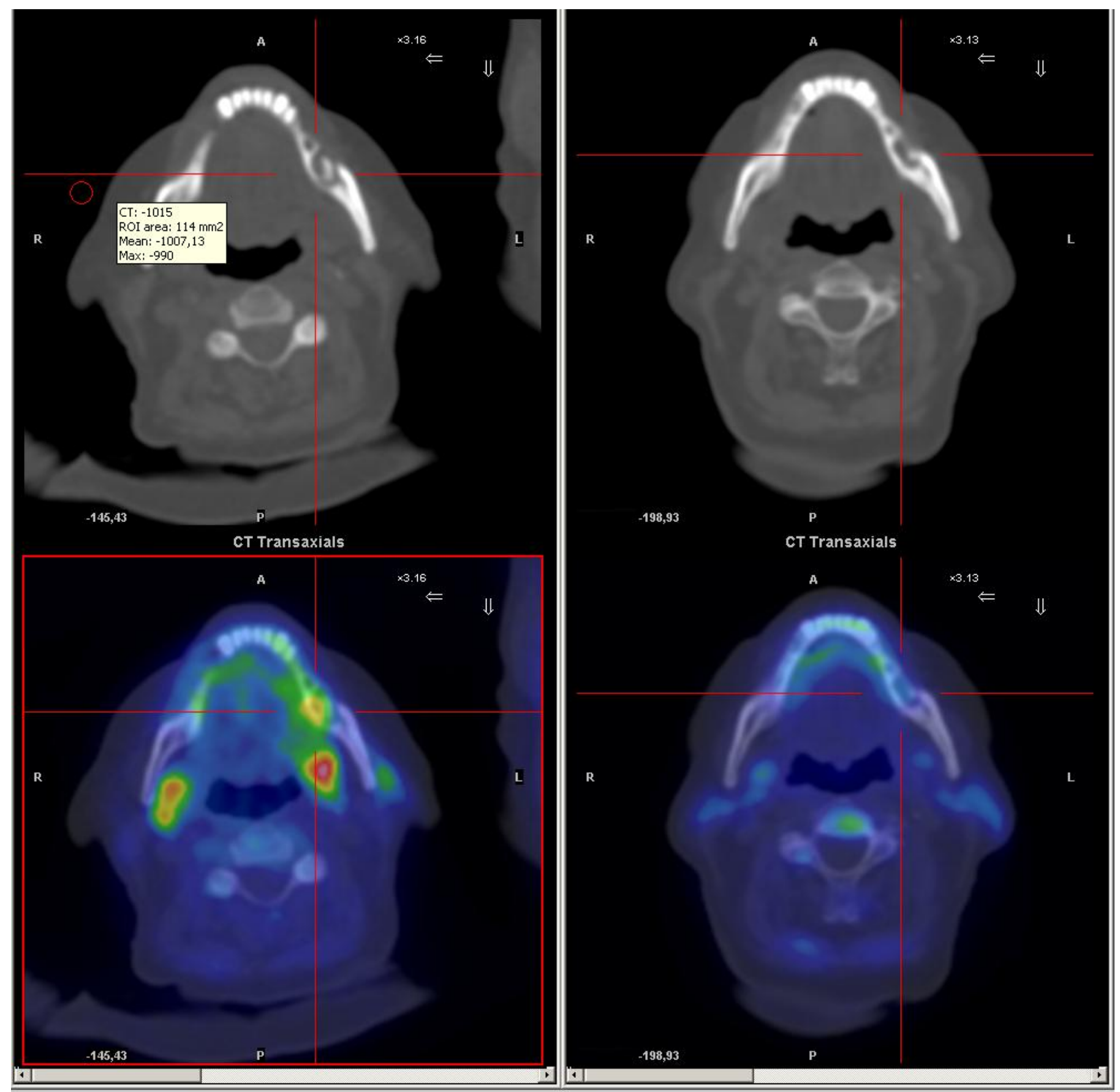

Fig 3 - ONJ site at two CT and PET-CT scans, 6 months before ONJ diagnosis (left) and two years later (right).

Case 3. 65 years, mandible ONJ after zoledronic acid treatment; no PET uptake was present at the ONJ site, at the exam performed 4 years after the ONJ diagnosis .

Case 4. 62 years, treated with both zoledronic acid and denosumab; maxillary and mandible multiple ONJ sites (left maxilla; bilateral in mandible) were all evident at PET scan eight months before the clinical diagnosis of ONJ and evolved at the exam performed 5 months after the ONJ diagnosis (Fig 4 and 5). 


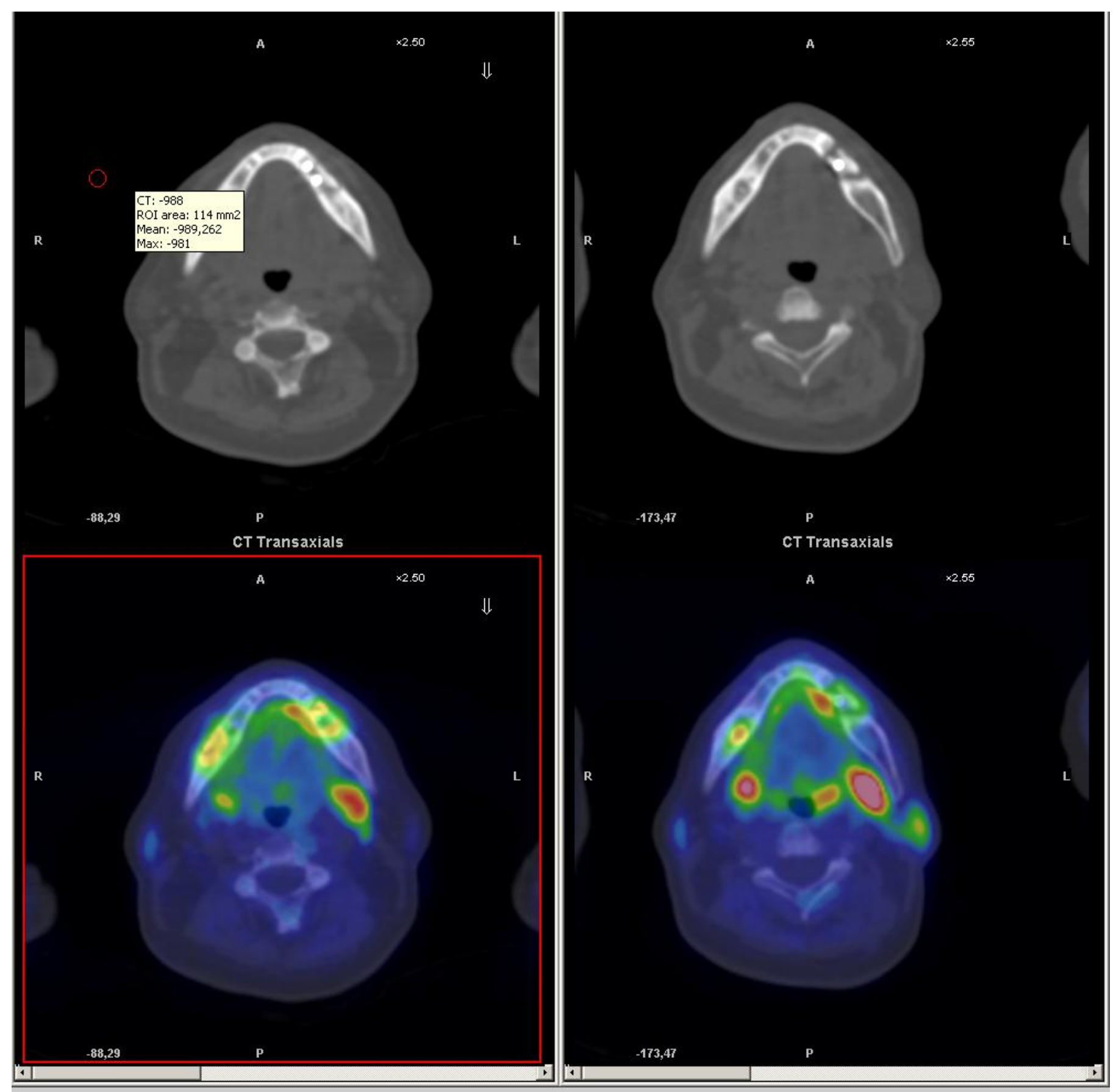

Fig 4. Evolution of CT scan and bilateral mandible ONJ uptakes, 8 months before ONJ diagnosis (left images) versus 5 months after ONJ diagnosis (right images). 


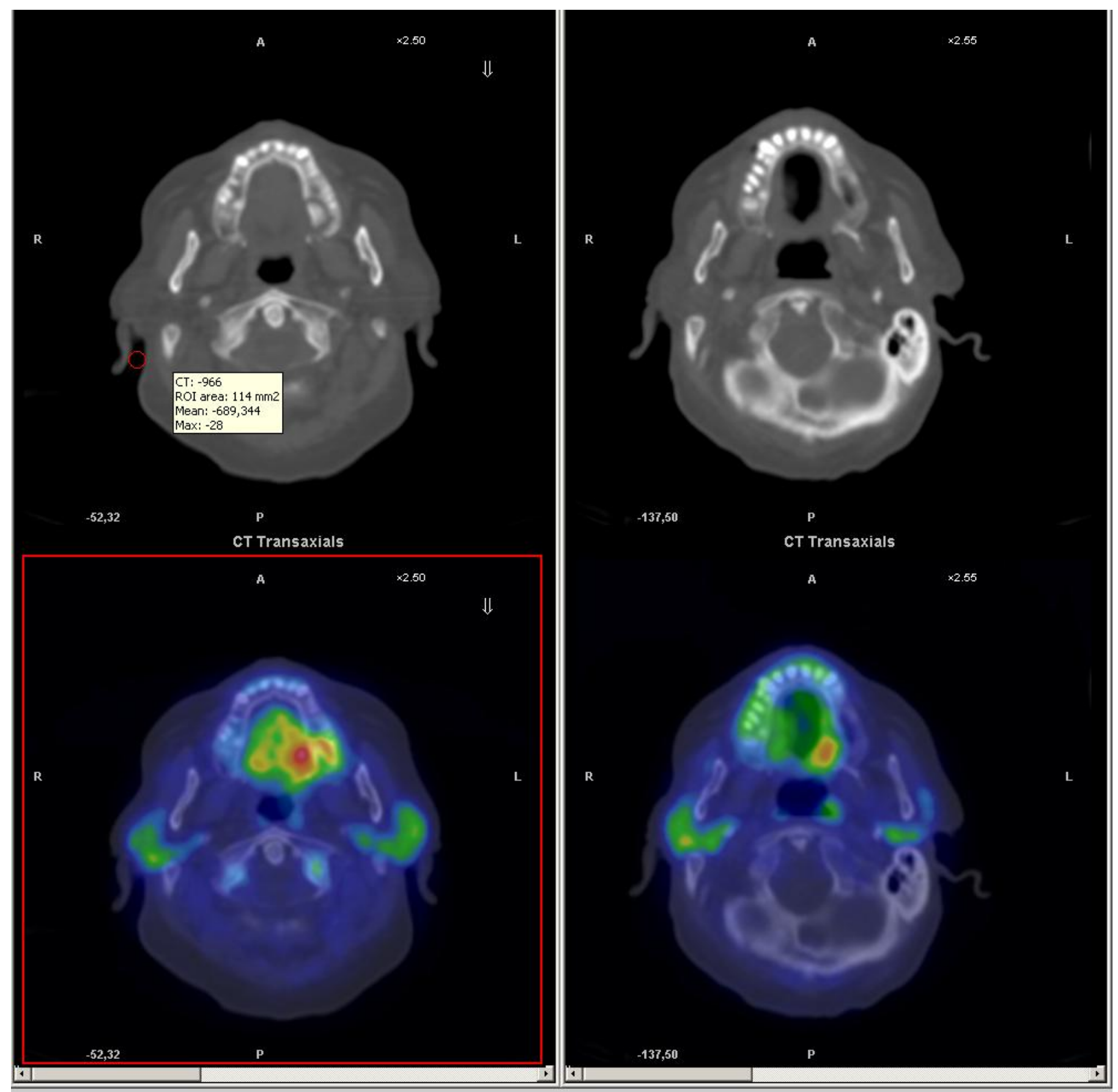

Fig 5. Evolution of CT scan and left maxilla ONJ uptake, 8 months before ONJ diagnosis (left images) versus 5 months after ONJ diagnosis (right images).

Case 5. 78 years, treated with zoledronic acid; mandible ONJ; you can see uptake at the PET performed one month before the ONJ diagnosis. (Fig 6). 


\section{Patient Id: 1027083}

Gems

Nuclear Imaging Environment

$-66,10$

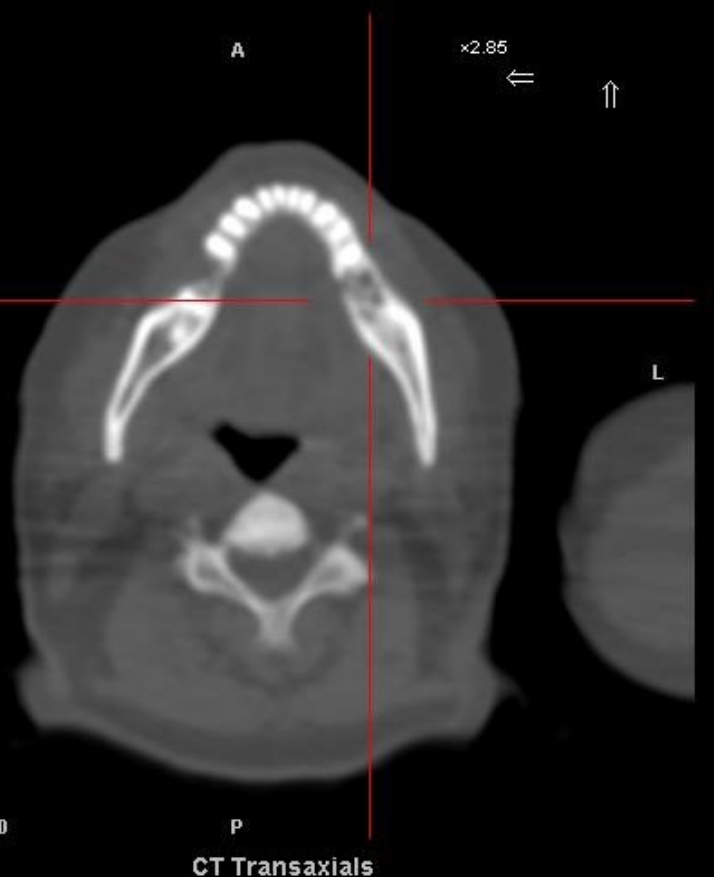

A

R

$-66,10$

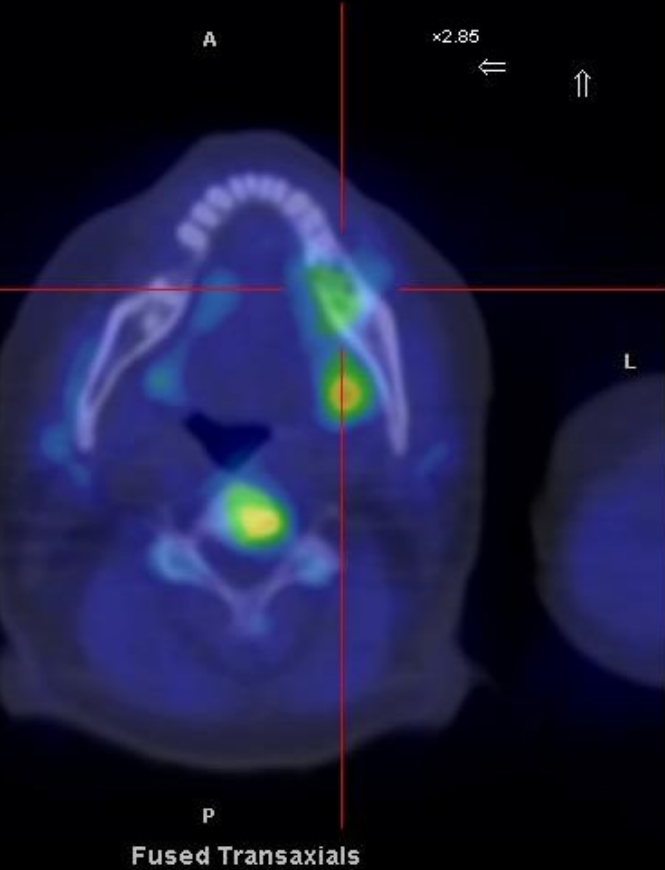

\section{Date \& Time: $21 / 04 / 2016$}

Manufacturer Model: Discovery 600

A

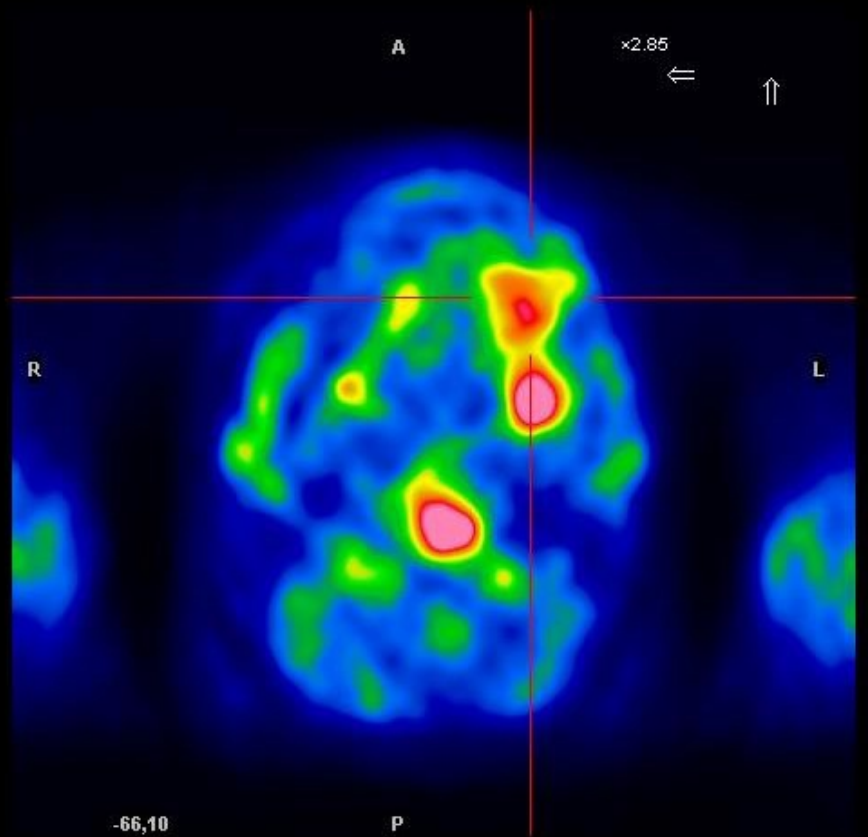

PET Transaxials
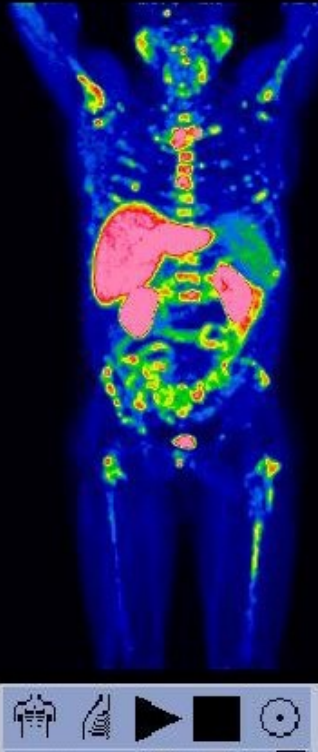

>116อ2

Fig 6. Mandible uptake next to ONJ diagnosis.

MIP Navigate

Conclusions. Our experience is very limited and needs studies with larger numbers of prostate cancer patients, with and without ONJ disease, mightily in several different centres. However our preliminary results indicate that 18F-Choline PETCT scan uptakes, as well as planar bone scan uptakes, are of potential help to early detect ONJ areas, both in presence and in absence of signs and symptoms. 
In our opinion, the 18F-Choline PET-CT scan uptakes at maxillary bone and mandible should be reported and underlined by Nuclear Medicine specialists, as well as bone scan uptakes, as an aid to oncologists, oral care specialists and patients.

\section{References}

1. ^A. Muni, V. Fusco, H. Rouhanifar, B. Greco, and L. Tommasi. (2014). Adjunctive role of bone scan (TC 99 scintigraphy) for diagnosis of exposed and unexposed osteonecrosis of jaw (ONJ). Ann Stomatol (Roma). 2014 Apr-Jun; 5(2 Suppl): 25. Published online 2014 May 9.PMCID: PMC4377699.

2. ${ }^{a}$ b V. Fusco, A. Muni, H. Rouhanifar, B. Greco, and L. Tommasi. (2014). Is bone scan (Tc99 scintigraphy) uptake predictive of clinical onset of osteonecrosis of jaw (ONJ)? Ann Stomatol (Roma). 2014 Apr-Jun; 5(2 Suppl): 11. Published online 2014 May 9. PMCID: PMC4377671.

3. ^ F. Dore, L. Filippi, M. Biasotto, S. Chiandussi, et al. (2008). Bone Scintigraphy and SPECT/CT of BisphosphonateInduced Osteonecrosis of the Jaw. Journal of Nuclear Medicine, vol. 50 (1), 30-35. doi:10.2967/jnumed.107.048785.

4. ^Frank Wilde, Karen Steinhoff, Bernhard Frerich, Thomas Schulz, et al. (2009). Positron-emission tomography imaging in the diagnosis of bisphosphonate-related osteonecrosis of the jaw. Oral Surgery, Oral Medicine, Oral Pathology, Oral Radiology, and Endodontology, vol. 107 (3), 412-419. doi:10.1016/.tripleo.2008.09.019.

5. ^Felice S. O'Ryan, Sam Khoury, Wendy Liao, Myo M. Han, et al. (2009). Intravenous Bisphosphonate-Related Osteonecrosis of the Jaw: Bone Scintigraphy as an Early Indicator. Journal of Oral and Maxillofacial Surgery, vol. 67 (7), 1363-1372. doi:10.1016/j.joms.2009.03.005.

6. ^R. Guggenberger, D.R. Fischer, P. Metzler, G. Andreisek, et al. (2012). Bisphosphonate-Induced Osteonecrosis of the Jaw: Comparison of Disease Extent on Contrast-Enhanced MR Imaging. [18F] Fluoride PET/CT, and Conebeam CT imaging. AJNR Am J Neuroradiol, vol. 34 (6), 1242-1247. doi:10.3174/ajnr.a3355.

7. ^Ryan Belcher, Jennings Boyette, Tiffany Pierson, Eric Siegel, et al. (2014). What Is the Role of Positron Emission Tomography in Osteonecrosis of the Jaws?. Journal of Oral and Maxillofacial Surgery, vol. 72 (2), 306-310. doi:10.1016/j.joms.2013.07.038.

8. `Kenneth E. Fleisher, Roy A. Raad, Rajan Rakheja, Vikas Gupta, et al. (2014). Fluorodeoxyglucose Positron Emission Tomography With Computed Tomography Detects Greater Metabolic Changes That Are Not Represented by Plain Radiography for Patients With Osteonecrosis of the Jaw. Journal of Oral and Maxillofacial Surgery, vol. 72 (10), 1957 1965. doi:10.1016/j.joms.2014.04.017.

9. ^H. Miyashita, H. Shiba, H. Kawana, T. Nakahara. (2015). Clinical utility of three-dimensional SPECT/CT imaging as a guide for the resection of medication-related osteonecrosis of the jaw. International Journal of Oral and Maxillofacial Surgery, vol. 44 (9), 1106-1109. doi:10.1016/j.ijom.2015.05.002.

10. ^Alexandre T. Assaf, Tomislav A. Zrnc, Chressen C. Remus, Gerhard Adam, et al. (2015). Intraindividual comparison of preoperative 99mTc-MDP SPECT/CT and intraoperative and histopathological findings in patients with 
bisphosphonate- or denosumab-related osteonecrosis of the jaw. Journal of Cranio-Maxillofacial Surgery, vol. 43 (8), 1461-1469. doi:10.1016/j.jcms.2015.06.025.

11. ${ }^{a, b}$ Christian Thomas, Magdalena Spanidis, Christina Engel, Frederik C. Roos, et al. (2015). Bone scintigraphy predicts bisphosphonate-induced osteonecrosis of the jaw (BRONJ) in patients with metastatic castration-resistant prostate cancer (mCRPC). Clin Oral Invest, vol. 20 (4), 753-758. doi:10.1007/s00784-015-1563-8.

12. `Yemi Kim, Ho-Young Lee, Hai-Jeon Yoon, Bom Sahn Kim. (2016). Utility of 18F-fluorodeoxy glucose and 18F-sodium fluoride positron emission tomography/computed tomography in the diagnosis of medication-related osteonecrosis of the jaw: A preclinical study in a rat model. Journal of Cranio-Maxillofacial Surgery, vol. 44 (4), 357-363. doi:10.1016/j.jcms.2016.01.009.

13. `Satoru Watanabe, Kenichi Nakajima, Atsushi Mizokami, Hiroshi Yaegashi, et al. (2016). Bone scan index of the jaw: a new approach for evaluating early-stage anti-resorptive agents-related osteonecrosis. Ann Nucl Med, vol. 31 (3), 201 210. doi:10.1007/s12149-016-1145-0.

14. 'Satoru Watanabe, Kenichi Nakajima, Seigo Kinuya. (2019). „ـ Symposium: Imaging modalities for drug-related osteonecrosis of the jaw (5), utility of bone scintigraphy and 18F-FDG PET/CT in early detection and risk assessment of medication-related osteonecrosis of the jaw (secondary publication). Japanese Dental Science Review, vol. 55 (1), 76-79. doi:10.1016/j.jdsr.2018.12.002.

15. ^Hidetaka Miyashita, Kaori Kameyama, Mayu Morita, Taneaki Nakagawa, et al. (2019). Three-dimensional radiologicpathologic correlation of medication-related osteonecrosis of the jaw using 3D bone SPECT/CT imaging. Dentomaxillofacial Radiology, vol. 48 (8), 20190208. doi:10.1259/dmfr.20190208.

16. ^IChiro Ogura, Eizaburo Kobayashi, Ken Nakahara, Kensuke Igarashi, et al. (2019). Quantitative SPECT/CT imaging for medication-related osteonecrosis of the jaw: a preliminary study using volume-based parameters, comparison with chronic osteomyelitis. Ann Nucl Med, vol. 33 (10), 776-782. doi:10.1007/s12149-019-01390-5.

17. 'I. Ogura, Y. Sasaki, M. Sue, T. Oda, et al. (2020). Tc-99m hydroxymethylene diphosphonate SPECT/CT for the evaluation of osteonecrosis of the jaw: preliminary study on diagnostic ability of maximum standardised uptake value. Clinical Radiology, vol. 75 (1), 46-50. doi:10.1016/j.crad.2019.05.025.

18. ${ }^{a}$, b Alberto Bedogni, Giuseppina Campisi, Vittorio Fusco, Alessandro Agrillo. (2013). Raccomandazioni clinicoterapeutiche sull'osteonecrosi delle ossa mascellari associata a bisfosfonati e sua prevenzione. Cleup.

19. a, biuseppina Campisi Alberto Bedogni Vittorio Fusco. (2020). Raccomandazioni clinico-terapeutiche sull'osteonecrosi delle ossa mascellari (ONJ) farmaco-relata e sua prevenzione. Unipapress.

20. ^Alberto Bedogni, Stefano Fedele, Giorgio Bedogni, Matteo Scoletta, et al. (2014). Staging of osteonecrosis of the jaw requires computed tomography for accurate definition of the extent of bony disease. British Journal of Oral and Maxillofacial Surgery, vol. 52 (7), 603-608. doi:10.1016/j.bjoms.2014.04.009.

21. 'Stefano Fedele, Giorgio Bedogni, Matteo Scoletta, Gianfranco Favia, et al. (2015). Up to a quarter of patients with osteonecrosis of the jaw associated with antiresorptive agents remain undiagnosed. British Journal of Oral and Maxillofacial Surgery, vol. 53 (1), 13-17. doi:10.1016/j.bjoms.2014.09.001.

22. ' L. Tommasi ", A. Fasciolo, V. Fusco, L. Benzi, A. Muni. (2018). Role of nuclear medicine imaging techniques in early diagnosis of ONJ in prostate cancer patients: report of a demonstrative case. Minerva Stomatologica 67 suppl 3 (36). 
